\title{
Data-driven approach for evaluation of formation damage during the injection process
}

\author{
Ali Shabani ${ }^{1} \cdot$ Hamid Reza Jahangiri $^{2} \cdot$ Abbas Shahrabadi $^{3}$
}

Received: 21 June 2019 / Accepted: 18 August 2019 / Published online: 24 August 2019

(c) The Author(s) 2019

\begin{abstract}
Waterflooding is among the most common oil recovery methods which is implemented in the most of oil-producing countries. The goal of a waterflooding operation is pushing the low-pressure remained oil of reservoir toward the producer wells to enhance the oil recovery factor. One of the important objects of a waterflooding operation management is understanding the quality of connection between the injectors and the producers of the reservoir. Capacitance resistance model (CRM) is a data-driven method which can estimate the production rate of each producer and the connectivity factor between each pair of wells, by history matching of the injection and production data. The estimated connectivity factor can be used for understanding the quality of connection between the wells. In the waterflooding operation, the injected water always has the potential of causing formation damage by invasion of foreign particles deep bed filtration (DBF), mobilization of indigenous particles (fines migration), scale formation, etc. The formation damage can weaken the quality of connection (connectivity factor), between the injectors and producers of the field, increasing the skin of injection well. In this paper, DBF is used for creation of formation damage in synthetic reservoir models. Then, it has been tried to find the existence and amount of formation damage by evaluating the connectivity factor of CRM. Finally, the results of that have been used for prediction of skin variation in a real case by using the connectivity factor of CRM.
\end{abstract}

Keywords CRM $\cdot$ Connectivity factor $\cdot \mathrm{DBF} \cdot$ Formation damage $\cdot$ Skin

\section{List of symbols}

$C_{\mathrm{p}} \quad$ Concentration of particles in the carrier fluid $(\mathrm{g} / \mathrm{L})$

$C_{\mathrm{o}} \quad$ Compressibility of oil $(1 / \mathrm{Pa})$

$C_{\mathrm{w}} \quad$ Compressibility of water $(1 / \mathrm{Pa})$

$f \quad$ Fraction of the inlet rate that is directed toward the outlet rate in CRM (connectivity factor)

$i \quad$ Injection rate $\left(\mathrm{m}^{3} /\right.$ day)

$J \quad$ Productivity index of the producer $\left(\mathrm{m}^{3} /\right.$ day $\left.\mathrm{Pa}\right)$

$K \quad$ Permeability of the medium $\left(\mathrm{m}^{2}\right)$

$K_{\text {o }} \quad$ Initial average permeability of the medium $\left(\mathrm{m}^{2}\right)$

$K_{\text {ro }} \quad$ Relative permeability of oil

$K_{\mathrm{rw}} \quad$ Relative permeability of water

Ali Shabani

alishabani.sharif@gmail.com

1 Department of Chemical and Petroleum Engineering, Sharif University of Technology, Tehran, Iran

2 Department of Chemical and Petroleum Engineering, Iran University of Science and Technology, Tehran, Iran

3 Research Institute of Petroleum Industry (RIPI), West Blvd. of Azadi Sports Complex, Tehran, Iran
$\left(K_{\mathrm{ro}}\right)_{S_{\mathrm{wc}}} \quad$ Relative permeability of oil at $S_{\mathrm{wc}}$

$\left(K_{\mathrm{rw}}\right)_{S_{\text {or }}} \quad$ Relative permeability of water at $S_{\text {or }}$

$n_{\mathrm{o}}$

$n_{\mathrm{w}} \quad$ Exponent of relative permeability curve of water

$P \quad$ Pressure $(\mathrm{Pa})$

$P_{\text {wf }} \quad$ Wellbore pressure of the producer $(\mathrm{Pa})$

$q \quad$ Production rate $\left(\mathrm{m}^{3} /\right.$ day)

$S_{\text {o }} \quad$ Saturation of the oil

$S_{\mathrm{w}} \quad$ Saturation of the water

$T \quad$ Time in field scale (day)

$t \quad$ Time in core scale (s)

$U \quad$ Magnitude of the Darcy velocity in core scale $(\mathrm{m} / \mathrm{s})$

$U_{\mathrm{o}} \quad$ Darcy velocity of the oil (m/day)

$U_{\mathrm{w}} \quad$ Darcy velocity of the water ( $\mathrm{m} /$ day)

$\beta \quad$ Formation damage coefficient

$\lambda \quad$ Filtration coefficient $(1 / \mathrm{m})$

$\Delta \varphi_{\mathrm{m}} \quad$ Maximum porosity reduction

$\varphi \quad$ Porosity of the medium

$\varphi_{\mathrm{o}} \quad$ Initial average porosity of the medium

$\mu \quad$ Viscosity of the injected liquid (cp)

$\mu_{\mathrm{o}} \quad$ Viscosity of the oil (cp) 


$\begin{array}{ll}\mu_{\mathrm{w}} & \text { Viscosity of the water (cp) } \\ \rho_{\mathrm{p}} & \text { Density of particles (g/L) } \\ \tau & \text { Time constant }\end{array}$

\section{Introduction}

There are several methods for understanding the quality of connection between injectors and producers of a field, including tracers, traditional reservoir simulators and datadriven approaches (Moghadam et al. 2011; Rong et al. 2016; Suleymanov et al. 2016; Salehian and Çınar 2019). A datadriven approach uses the injection and production data of a field in order to find the quality of connection between the wells. Capacitance resistance model (CRM) is the most famous data-driven approach that has been introduced and developed recently (Sayarpour et al. 2009). Generally, a CRM can predict output signal of any input-output system that contains a certain mass, electrical charges or heat flow. In the petroleum industry, CRM enables a reservoir engineer to estimate the production rates of producers and also understand the quality of connection between each pair of injector and producer of the field, by history matching of the injection and production data (Sayarpour 2008; Sayarpour et al. 2009; Izgec and Kabir 2010; Kaviani et al. 2012; Moreno 2013; Parekh and Kabir 2013; Zhang et al. 2017; de Holanda et al. 2018; Nwachukwu et al. 2018). In a CRM, the quality of connection between the wells is achieved by evaluation of a matching parameter called connectivity factor (Sayarpour et al. 2009; Weber et al. 2009; Mirzayev et al. 2017).

During a waterflooding operation, the formation rock can be damaged by particle invasion, fines migration, minerals precipitation, and pore deformation or collapse (Liu and Civan 1996; Naseri et al. 2015; Vaz et al. 2016; Mahmoud et al. 2017; Kamal et al. 2019). Particles transport and capturing (DBF) and its effect on permeability of the porous medium are among the main reasons of formation damage (Todd et al. 1984; Baghdikian et al. 1987; Bedrikovetsky et al. 2001; Moghadasi et al. 2004; Wong and Mettananda 2010; Yuan and Shapiro 2010; Bennacer et al. 2013; Oliveira et al. 2014; Sacramento et al. 2015; Kanimozhi et al. 2019). A particle in porous medium may be a part of the separated reservoir rock, a foreign particle entered to the reservoir by injected fluid or a scale that is generated in the reservoir due to the mixing of incompatible waters (Wat et al. 1992). When a particle is traveling through the pores, it may get stuck with one of the underlying mechanisms:

Pore plugging: Some of the particles may bridge or plug the pores when traveling through them as presented in Fig. 1a. These particles severely reduce the permeability of the porous medium and also reduce the porosity.

Surface deposition: Some other injected particles may deposit on the surface of the pores by mechanisms presented in Fig. 1b. These particles reduce the porosity and have a small effect on the permeability of the porous medium. Small-size particles may cross the pores without getting stuck.

Many researchers have tried to simulate DBF in a core scale using the continuity equation of the particles (Herzig et al. 1970; Bedrikovetsky et al. 2006; Alem et al. 2013; You et al. 2014; Sacramento et al. 2015). These researchers have tried to match their simulation with experimental data by estimation of proper matching parameters. The main challenge in development of DBF equations and its application for the flooding operation is the correct determination of the particles capturing rate and its impact on the permeability of the system. The equation of the particles capturing rate used by most of the researchers is as presented below (Iwasaki et al. 1937; Herzig et al. 1970; Vaz et al. 2017):

$$
\frac{\partial \sigma}{\partial t}=\lambda U C_{\mathrm{p}}
$$

Fig. 1 Main mechanisms of the particle capturing in the porous media (Vaz et al. 2017)

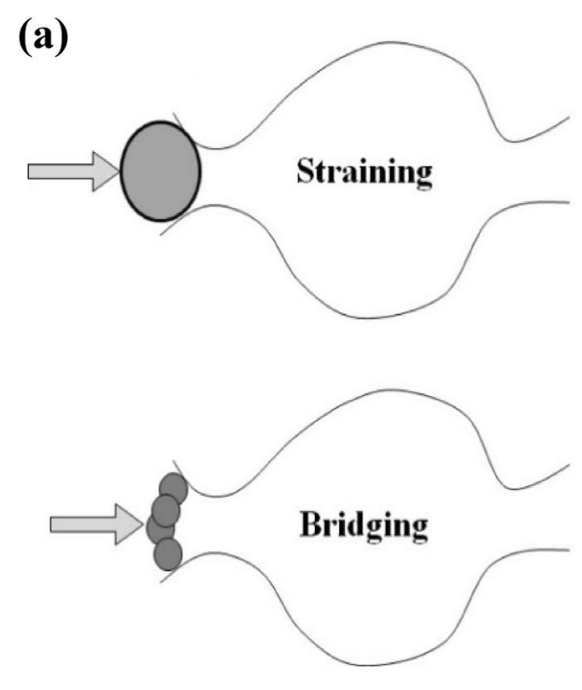

(b)

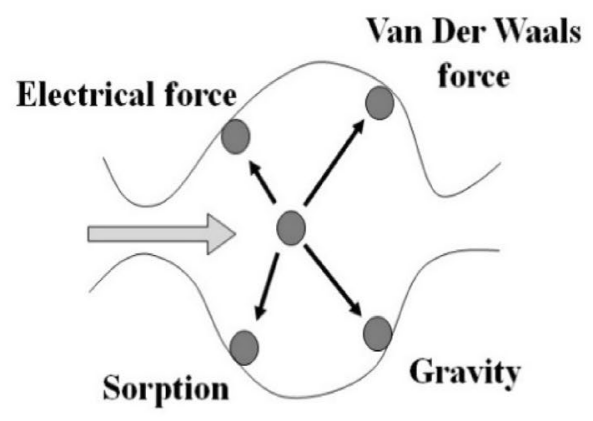


In this equation, $\lambda$ is filtration coefficient, which determines the particles capturing rate (the transport rate of the particles from the bulk to the rock vacant pores). Actually, this parameter determines the particle deposition profile along the porous medium. As amount of the filtration coefficient increases, the particles tend to deposit in the injection point of system. Based on different definition of the filtration coefficients, different DBF models can be developed. Some researchers tried to involve the effect of hydrodynamic in the continuity equations of particles. For example, Yuan and Shapiro (2011) considered the effect of detachment of particles (due to the fluid velocity) after deposition and plugging (Yuan and Shapiro 2011). This phenomenon has been involved in asphaltene precipitation and deposition models too (Wang and Civan 2001). Yuan and Shapiro (2011) presented a model for non-monotonic deposition profiles by definition of the inverse filtration coefficients (transport of particles from the rock surface to the fluid bulk). Alem et al. (2013) considered the effect of hydrodynamic force (fluid velocity) on the filtration coefficient and showed that the low injection velocity enhances the deposition of particles (Alem et al. 2013).

Most of the researchers have used the underlying equation (Sharma et al. 2000; Bedrikovetsky et al. 2006) or a similar equation with extra matching parameters (AlAbduwani et al. 2005; Bedrikovetsky et al. 2011; Vaz et al. 2017) for determination of the permeability reduction due to the particles deposition.

$K=\frac{K_{0}}{1+\beta \sigma}$

where $K_{0}$ is the initial permeability and $\beta$ is the formation damage factor. There are researchers that have tried to involve pore and particle size distribution in continuity equation of particles in order to present a stochastic model for DBF (Bedrikovetsky et al. 2003; Santos and Bedrikovetsky 2006; Yuan et al. 2013). Some researchers have studied particle transport mechanism in pore scale using pore network model (Todd et al. 1984; Sharma and Yortsos 1987; Rege and Fogler 1988; Yang and Balhoff 2017). Bedrikovetsky et al. (2011) applied the continuity equations of particles in the reservoir scale for determination of wellbore injectivity decline (Bedrikovetsky et al. 2011).

Invasion of foreign particles, mobilization of indigenous particles and formation of scale can create formation damage during a waterflooding operation (Moghadasi et al. 2004). It is believed that the formation damage can weaken the quality of connection between the injectors and producers of a reservoir. In the present paper, it is tried to understand the existence and the amount of formation damage (skin) from the results of a CRM. For this purpose, numerical simulation of DBF is used for creation of formation damage in the injector. The structure of the paper is as follows: Firstly, CRM and DBF models are presented, respectively. Secondly, it is tried to verify the DBF model by using the experimental data. Finally, it is tried to survey the effect of formation damage on the connectivity factor of a CRM, by using synthetic and real field data.

\section{Mathematical models}

\section{Capacitance resistance model}

By assuming different control volumes, different forms of the CRM can be developed, including CRMT, CRMP, and CRMIP (Sayarpour 2008; Weber et al. 2009; Salehian and Çınar 2019). CRMT (CRM Tank) is used for a system of one injector and one producer. But it also can be applied for a system of several injectors and several producers by assuming a pseudo injector and pseudo producer (Sayarpour 2008). In CRMT, by assuming a constant productivity index $(J)$ and a step variation of the injection rate and the producer pressure in the time interval of $\Delta t_{k}$, the producer rate can be estimated as presented below (Sayarpour 2008):

$q\left(t_{k}\right)=q\left(t_{k-1}\right) \mathrm{e}^{-\left(\frac{\Delta t_{k}}{\tau}\right)}+f\left(1-\mathrm{e}^{-\left(\frac{\Delta t_{k}}{\tau}\right)}\right)\left[i^{k}+J \tau \frac{\Delta P_{\mathrm{wf}}^{k}}{\Delta t_{k}}\right]$

where $q$ is the production rate of producer, $\tau$ is the time constant, $f$ is the fraction of injection volume that is toward the producer (connectivity factor; $0 \leq f \leq 1$ ), $i$ is the injection rate, $J$ is the productivity index, and $P_{\mathrm{wf}}$ is the pressure of producer.

CRM producer (CRMP) is used for multiple well systems. It estimates the production rate of each producer and the quality of connection (connectivity factor) between each pair of injector and producer. By assuming a constant productivity index and a step variation of the injection rate and producer pressure in the time interval of $\Delta t_{k}$, the production rate of each producer can be estimated as presented in Eq. 2 (Sayarpour 2008):

$q_{j}\left(t_{k}\right)=q_{j}\left(t_{k-1}\right) \mathrm{e}^{-\frac{\Delta t_{k}}{\tau_{j}}}+\left(1-\mathrm{e}^{-\frac{\Delta t_{k}}{\tau_{j}}}\right)\left[\sum_{i=1}^{N_{\mathrm{inj}}} f_{i j} I_{i}^{k}-J_{j} \tau_{j} \frac{\Delta P_{\mathrm{wf}, j}^{k}}{\Delta t_{k}}\right]$

In this equation, $q_{j}, \tau_{j}$, and $J_{j}$ are the production rate, the time constant, and the productivity index of the $j$ th producer, respectively. $f_{i j}$ is the connectivity factor between the $i$ th injector and the $j$ th producer, $I_{i}$ is the injection rate of the $i$ th injector, and $N_{\text {inj }}$ is the number of active injectors. Usually, the last term of Eqs. 1 and 2 is neglected (due to the small variation of the producer pressure). The connectivity

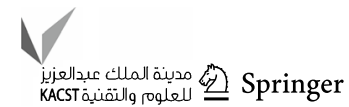


factor $\left(f_{i j}\right)$, time constant $\left(\tau_{j}\right)$, and the primary production rate of the producer $\left(q_{0}\right)$ are estimated using an optimization process. Among these parameters, the connectivity factor is used for determination of the quality of the connection between the injectors and the producers. It depends on transportation properties of formation rock and fluid. For example, a bigger amount of a connectivity factor in comparison with others may be due to a higher permeability or existence of a fracture between the wells (Kaviani et al. 2012). It is obvious that the sum of the connectivity factors for an injector must be less than one as presented below (Sayarpour et al. 2009):

$$
\sum_{i=1}^{N_{\text {inj }}} f_{i} \leq 1
$$

In the next sections, the DBF models are presented. Firstly, the equations of DBF for the single -phase fluid flow in the core scale are introduced (DBF in core scale). Good match between experiments and simulations shows that this model can properly model the permeability decline in the core flooding experiments. Then, this model is used for modeling of permeability decline in the field scale by two-phase fluid flow equations (DBF in field scale).

\section{DBF in core scale}

The equations of DBF for the single-phase fluid flow in the core scale are as presented below (Bedrikovetsky et al. 2006):

$$
\begin{aligned}
& U=-\frac{987 K}{\mu} \frac{\mathrm{d} P}{\mathrm{~d} x} \\
& \frac{\mathrm{d} \varphi}{\mathrm{d} t}+\frac{\mathrm{d} U}{\mathrm{~d} x}=0 \\
& \frac{\mathrm{d}\left(\varphi C_{\mathrm{p}}\right)}{\mathrm{d} t}+\frac{\mathrm{d}\left(U C_{\mathrm{p}}\right)}{\mathrm{d} x}+\lambda_{0}\left(1-\frac{\varphi_{0}-\varphi}{\Delta \varphi_{\mathrm{m}}}\right) U C_{\mathrm{p}}=0 \\
& \frac{\mathrm{d} \varphi}{\mathrm{d} t}=\frac{\varphi \lambda_{0}\left(1-\frac{\varphi_{0}-\varphi}{\Delta \varphi_{\mathrm{m}}}\right) U C_{\mathrm{p}}}{\rho_{\mathrm{p}}} \\
& K=\frac{K_{0}}{1+\beta\left(\varphi_{0}-\varphi\right)}
\end{aligned}
$$

In these equations, $K$ is the permeability of the medium, $\mu$ is the viscosity of the fluid, $P$ is the pressure, $\varphi_{0}$ is the primary porosity, $\lambda_{0}$ is the initial filtration coefficient, and $\Delta \varphi_{\mathrm{m}}$ is the maximum reduction in the porosity of the medium $\left(\lambda_{0}, \Delta \varphi_{\mathrm{m}}\right.$, and $\beta$ are the matching parameters of the DBF model). Equation 5 is the continuity equation of the carrier fluid. The first two terms of Eq. 6 represent the accumulation and the convection of the particles, respectively. The last term of Eq. 6 represents the particles capturing rate (Herzig et al. 1970; Bedrikovetsky et al. 2006). Equation 7 relates the particles capturing rate to the porosity reduction. Equation 8 represents the effect of the porosity reduction on the permeability of system (Pang and Sharma 1997; Sharma et al. 2000; Bedrikovetsky et al. 2006).

As mentioned earlier, some injected particles may deposit over the surface of pores by surface deposition mechanisms. These deposited particles do not change the permeability considerably. But still there is a chance for some of the deposited particles to roll and move on the surface of pores (Johnson et al. 2007). So a huge amount of particles (rolling particles with those moving in the bulk of fluid) are moving until they get stuck and plug the pores in the tight portions by pore plugging mechanism, as shown in Fig. 1a. Velocity of the carrier fluid $(U)$ has a direct impact on pore plugging mechanisms (Alem et al. 2013), so the fluid velocity must be involved in the rate of particles capturing rate as shown in the last term of Eq. 5. In this term, there is a parameter called maximum porosity reduction $\left(\Delta \varphi_{\mathrm{m}}\right)$. When the porosity change $\left(\varphi_{0}-\varphi\right)$ reaches its limit value $\left(\Delta \varphi_{\mathrm{m}}\right)$, the particles capturing rate gets zero and no more porosity reduction happens. This note origins from the idea that the big pores never get plugged during the filtration of particles.

When incompatible water is injected into the formation (for example, sulfated water), the ions of the injected water may interact with the ions of the formation water (for example, barium and strontium), and a mineral is formed (barite or celestite). The formed mineral may reduce the porosity and permeability as a particle, by deposition or plugging mechanisms. Hence, the modeling of DBF can cover the effect of scale precipitation-deposition too, and this is why the DBF was selected as the representation of the formation damage mechanisms in this study.

\section{DBF in field scale}

Equations of deep bed filtration for a two-phase fluid flow are based on the following assumptions:

- No particle can enter into the oil phase because the particles are water wet.

- Oil and water are slightly compressible fluids (with constant compressibility).

- The reservoir is homogenous.

- During flooding the viscosity of oil and water are constants.

- Capillary pressure is zero. 


$$
\begin{aligned}
& \frac{\mathrm{d}\left(\varphi S_{\mathrm{o}}\right)}{\mathrm{d} T}+\varphi S_{\mathrm{o}} C_{\mathrm{o}} \frac{\mathrm{d} P}{\mathrm{~d} T}+\frac{\mathrm{d} U_{\mathrm{o}}}{\mathrm{d} x}+\frac{Q_{\mathrm{o}}}{V}=0 \\
& \frac{\mathrm{d}\left(\varphi S_{\mathrm{w}}\right)}{\mathrm{d} T}+\varphi S_{\mathrm{w}} C_{\mathrm{w}} \frac{\mathrm{d} P}{\mathrm{~d} T}+\frac{\mathrm{d} U_{\mathrm{w}}}{\mathrm{d} x}+\frac{Q_{\mathrm{w}}}{V}=0 \\
& \frac{\mathrm{d}\left(\varphi S_{\mathrm{w}} C_{\mathrm{p}}\right)}{\mathrm{d} T}+\frac{\mathrm{d}\left(U_{\mathrm{w}} C_{\mathrm{p}}\right)}{\mathrm{d} x}+\frac{Q_{\mathrm{w}} C_{\mathrm{p}}}{V}+\lambda_{0}\left(1-\frac{\varphi_{0}-\varphi}{\Delta \varphi_{\mathrm{m}}}\right) U_{\mathrm{w}} S_{\mathrm{w}} C_{\mathrm{p}}=0
\end{aligned}
$$

In these equations, $S_{\mathrm{o}}$ and $S_{\mathrm{w}}$ are the oil and water saturation, $C_{\mathrm{o}}$ and $C_{\mathrm{w}}$ are the oil and water compressibility, and $U_{\mathrm{o}}$ and $U_{\mathrm{w}}$ are the oil and water velocity, respectively. The last terms of Eqs. 8 and 9 represent the sink or source terms. Equation 9, 10, and 11 are, respectively, the continuity equations of the oil, water, and the suspended particles. The last term in Eq. 11 represents the particles capturing rate in the field scale. The porosity variation due to the particles capturing is estimated through Eq. 12. The relationship between the porosity and permeability is estimated from Eq. 8 .

$\frac{\mathrm{d} \varphi}{\mathrm{d} T}=\frac{\varphi \lambda_{0}\left(1-\frac{\varphi_{0}-\varphi}{\Delta \varphi_{\mathrm{m}}}\right) U_{\mathrm{w}} C_{\mathrm{p}}}{\rho_{\mathrm{p}}}$

The oil and water Darcy velocities are expressed as:

$U_{\mathrm{o}}=-\frac{85.277 \times 10^{6} K K_{\mathrm{ro}}}{\mu_{\mathrm{o}}} \frac{\mathrm{d} P}{\mathrm{~d} x}$

$U_{\mathrm{w}}=-\frac{85.277 \times 10^{6} K K_{\mathrm{rw}}}{\mu_{\mathrm{w}}} \frac{\mathrm{d} P}{\mathrm{~d} x}$

where form the Corey correlations we have (Kalantariasl et al. 2014):

$K_{\mathrm{ro}}=\left(K_{\mathrm{ro}}\right)_{S_{\mathrm{wc}}}\left(\frac{1-S_{\mathrm{w}}-S_{\mathrm{or}}}{1-S_{\mathrm{wc}}-S_{\mathrm{or}}}\right)^{n_{\mathrm{o}}}$
$K_{\mathrm{rw}}=\left(K_{\mathrm{rw}}\right)_{S_{\mathrm{or}}}\left(\frac{S_{\mathrm{w}}-S_{\mathrm{wc}}}{1-S_{\mathrm{wc}}-S_{\mathrm{or}}}\right)^{n_{\mathrm{w}}}$

In these equations, $S_{\mathrm{wc}}$ is the connate water saturation, $S_{\mathrm{or}}$ is the residual oil saturation, $\left(K_{\mathrm{ro}}\right)_{S_{\mathrm{wc}}}$ is the relative permeability of water at $S_{\mathrm{wc}},\left(K_{\mathrm{rw}}\right)_{S}$ is the relative permeability for oil at $S_{\mathrm{or}}$, and $n_{\mathrm{w}}$ and $n_{\mathrm{o}}$ are the powers.

\section{Results and discussion}

\section{DBF model verification with experimental data}

In this section, the experiments of Moghadasi et al. (2004) have been studied in order to prove the correctness of the model in the core scale. Moghadasi et al. (2004) studied the effects of suspension concentration, flow rate, particle size, and particles initially present in the porous media (Moghadasi et al. 2004). Moghadasi et al. (2004) injected 1000 ppm suspension of aluminum oxide particles $(7 \mu \mathrm{m}$ diameter) into an artificial core made from spherical glass beads. The permeability and the porosity of the medium were 159 Darcy and 0.38 , respectively. Two experiments were accomplished in this medium with the flow rates of $25 \mathrm{~mL} / \mathrm{min}$ (Experiment 1) and $50 \mathrm{~mL} / \mathrm{min}$ (Experiment 2). Figure 2 shows the results of simulation in the core scale for the both experiments of Moghadasi et al. (2004). As it is shown in Fig. 2, the results of simulation have a good agreement with the experimental data. The optimized parameters after the simulation of these experiments are presented in Table 1.

Amount of the $\beta$ parameter is the same in both of the Moghadasi et al. (2004) experiments. This is because of the same pore space properties in these experiments (Carageorgos et al. 2010). The low velocity in the first experiment of Moghadasi et al. (2004) performs bridging of particles that results in the higher rate of the particles capturing $\left(\lambda_{0}\right)$ in
Fig. 2 Results of the simulation in the core scale for the experiments of Moghadasi et al. (2004)

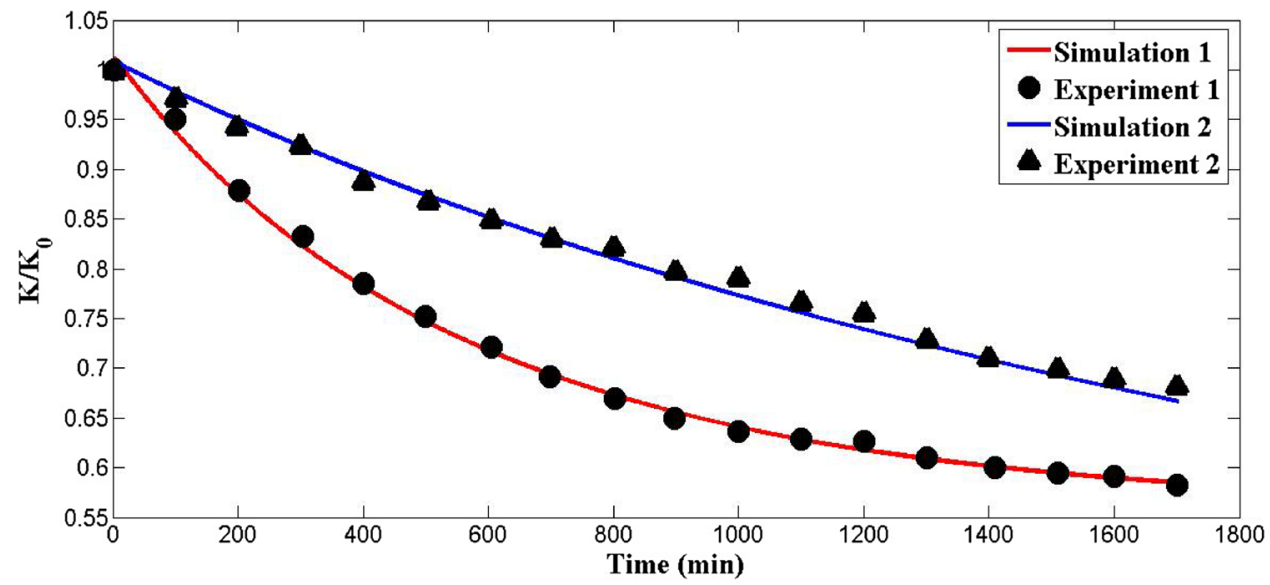


Table 1 Optimized parameters used for the simulation of the Moghadasi et al. (2004) experiments

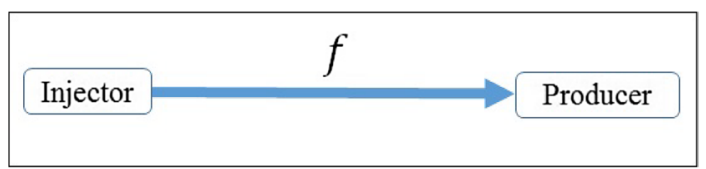

Fig. 3 The schematic of CRMT model in the synthetic case

Table 2 Parameters used in the 3 types of DBF simulations in the synthetic reservoir

\begin{tabular}{lrrr}
\hline Parameter & \multicolumn{1}{c}{$\beta$} & \multicolumn{1}{c}{$\lambda_{0}$} & $\Delta \varphi_{\mathrm{m}}$ \\
\hline No DBF & 0 & 0 & 0.5 \\
Medium DBF & 250 & 150 & 0.5 \\
Strong DBF & 500 & 150 & 0.5 \\
\hline
\end{tabular}

comparison with the second experiment (Alem et al. 2013). The lower rate of the particles capturing in the second experiment leads to the more vacant pores that are ready to get filled. Hence, the value of the maximum porosity reduction $\left(\Delta \varphi_{\mathrm{m}}\right)$ in the second experiment is bigger than the first one.

\section{Formation damage and CRM}

In order to study the effect of formation damage on CRM, a synthetic reservoir model has been developed using field scale DBF equations. In this synthetic model, there is one injector and one producer, so the CRMT equation has been applied for it (Eq. 1). $\tau, f$, and $q_{0}$ are the matching parameters of CRMT in the synthetic model, and are calculated from history matching of the injection and production data. In this model, the producer is working under a constant pressure, so during the optimization, the last term of Eq. 1 is ignored. The schematic of the CRMT model in the synthetic case is presented in Fig. 3. As mentioned before, the $f$ (achieved from optimization of Eq. 1) will be reported as the value of the connectivity factor.

According to the literature, the skin of a wellbore due to the formation damage is a positive number and less than 20 (Horne 1995). So, the matching parameters of the DBF model in field scale are adjusted to create the formation damage in this acceptable range. The model is run in 3 different DBF conditions including no formation damage (no deep bed filtration), medium formation damage (medium deep bed filtration), and severe formation damage (severe deep bed filtration). The parameters used in each of these runs are presented in Table 2. As it is presented in Table 2, the amount of $\lambda_{0}$ is bigger than the experimental data. The bigger value of $\lambda_{0}$ makes the formation damage in the vicinity of the injector.

In Table 3, the constants used for simulation of the synthetic case are presented. The field DBF equations (Eqs. 9-16) are solved numerically using fully implicit finite difference method. In the synthetic case, the injection rate is sinusoidal flocculated to observe the effect of lag time between injector and producer (Fig. 4). The production rates achieved from simulation (DBF model) and estimation (CRM) in three different conditions of formation damage are presented in Fig. 4. As it is shown in Fig. 4, CRM cannot match the production rate of a severe formation damage case properly, because formation damage is a time-dependent process.

In Fig. 5, the skin (achieved from permeability reduction of the DBF model) and connectivity factor (achieved from CRM) versus time are presented. As it was mentioned before, the formation damage is a time-dependent process, so as the time interval of the CRM is increasing the amount of the connectivity factor is decreasing. In other words, a continuous reduction of connectivity factor is due to the existence of a time-dependent formation damage.

In Fig. 6, the connectivity factor versus skin is presented for the two cases of medium and severe formation damage. As it is shown in Fig. 6, there is an almost linear relationship between theses parameters, when there is a formation damage in the system. This relationship can be

Table 3 Constants used for simulations of the synthetic reservoir

\begin{tabular}{llll}
\hline$K_{0}$ & $\varphi_{0}$ & $P_{\text {Initial }}$ & $P_{\text {Production }}$ \\
\hline 1 day & 0.15 & $3000 \mathrm{psi}$ & $2900 \mathrm{psi}$ \\
\hline$\mu_{\mathrm{w}}$ & $\mu_{\mathrm{o}}$ & $S_{\mathrm{wc}}$ & $S_{\text {or }}$ \\
\hline $1 \mathrm{cp}$ & $2 \mathrm{cp}$ & 0.2 & 0.1 \\
\hline$C_{\mathrm{w}}$ & $C_{\mathrm{o}}$ & $n_{\mathrm{w}}$ & $n_{\mathrm{o}}$ \\
\hline $10 \mathrm{e}-61 / \mathrm{Pa}$ & $5 \times 10 \mathrm{e}-61 / \mathrm{Pa}$ & 1.5 & 2.5 \\
\hline
\end{tabular}


Fig. 4 Simulated and estimated (CRM) production rate in different formation damage conditions

Fig. 5 Connectivity factor and skin versus time for the synthetic case

Fig. 6 Connectivity factor versus skin for the synthetic case
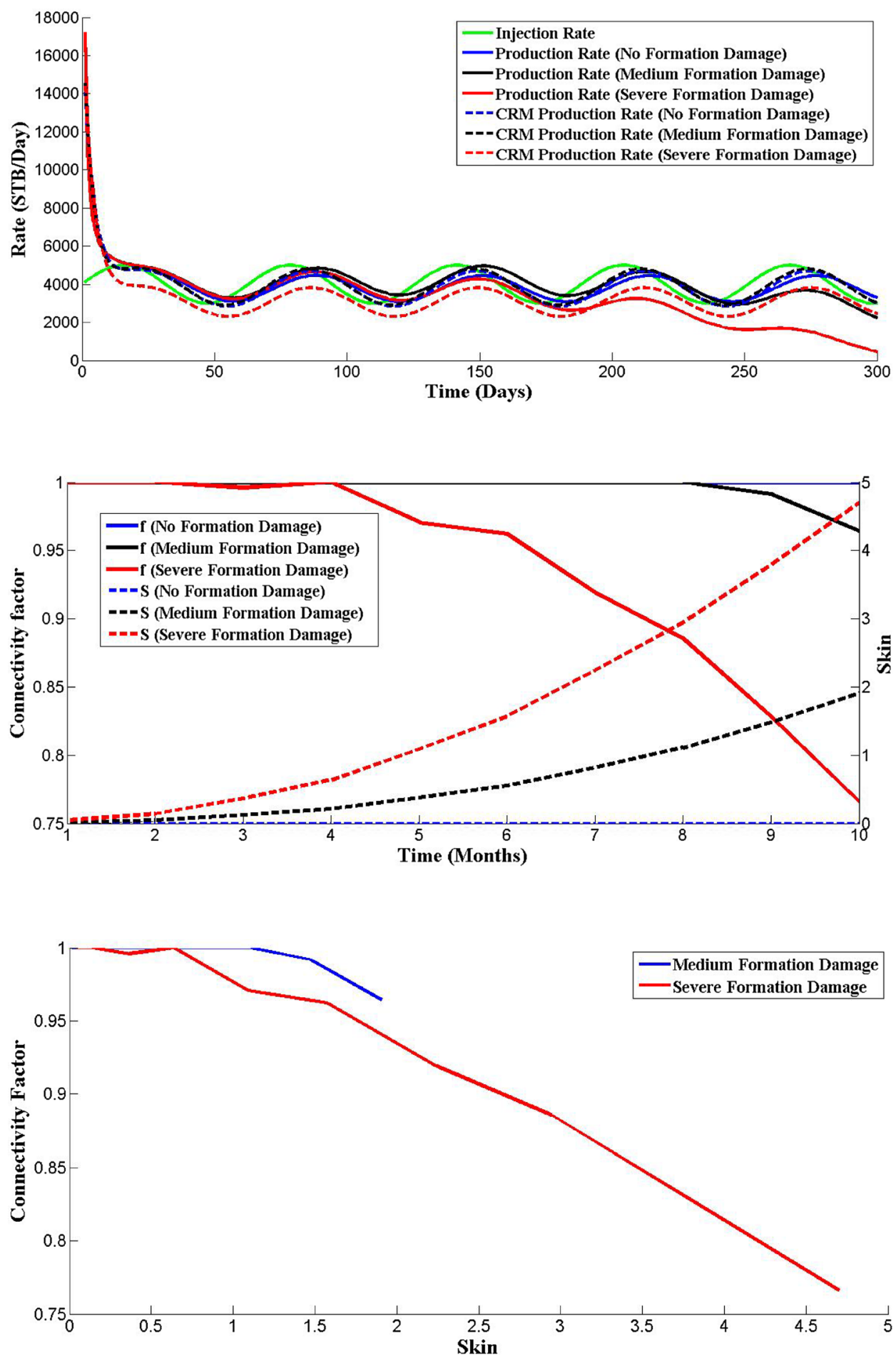

used for finding the skin from a set of connectivity factor data.

In the following, the most important parameters that may affect the results of a CRM-Formation damage model are discussed.

\section{Effect of injection rate pattern}

In a real injection operation, the injection rate may vary wild and randomly. Hence, a random injection rate pattern has been selected to study the effect of rate fluctuation as presented in Fig. 7. Note that the total volume of 
Fig. 7 Simulated production rate in a random injection rate pattern

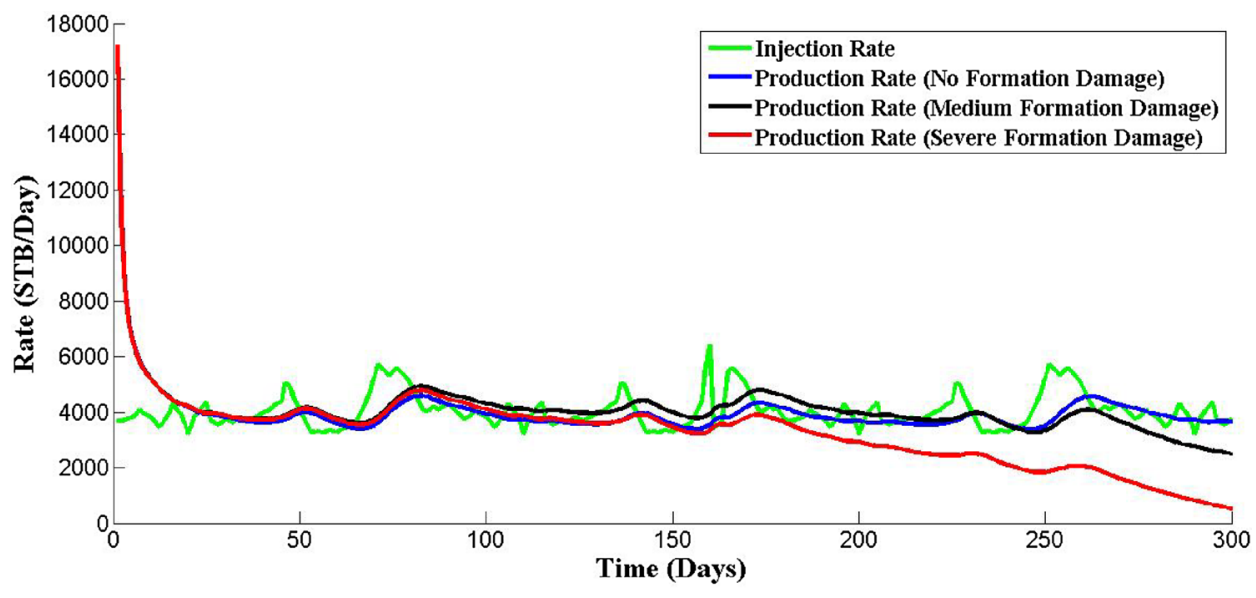

Fig. 8 Connectivity factor and skin versus time for a different injection rate pattern

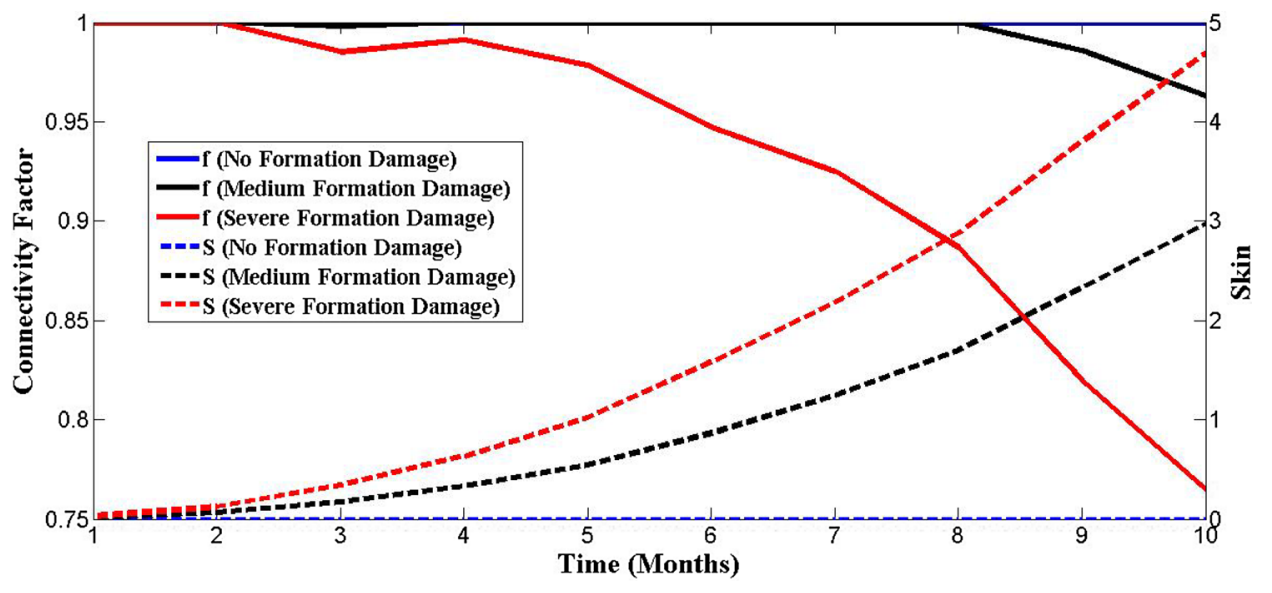

Effect of multi-well production

For understanding the effect of multi-well production, four producer wells were installed in four points of the synthetic reservoir. In this case, CRMP is used for calculation of the connectivity factor (Eqs. 2-3). The schematic of CRMP in the multi-production system is presented in Fig. 9. According to Fig. 9, the value of the injector connectivity factor is the sum of the connectivity factors between the injector and all of the producers $\left(f=\sum_{i=1}^{N_{\text {inj }}} f_{i}\right)$.

In Fig. 10, the connectivity factor and skin versus time for different number of production wells are presented. As it is shown in Fig. 10, the number of active production wells has no effect on connectivity factor, and a damaged case can easily be detected from a system of multi-production.

\section{Effect of heterogeneity}

In order to study the effect of heterogeneity, initial permeability and porosity of synthetic model's grids were adjusted by using normal distribution function. $20 \%, 10 \%$, and $0 \%$ variance were selected to create the heterogeneity in the 
model. In Fig. 11, the results of simulation for different heterogeneities (with no formation damage) are compared with the homogeneous synthetic case (with formation damage). The results of that show that in the early times of injection the trend of connectivity factor in the heterogeneous case reduces continuously, like something happened for the trend of connectivity factor in the homogeneous case. But after enough time, the connectivity factor does not change anymore in the heterogeneous cases. This stabilization of connectivity factor can be used as the criteria of difference between heterogeneity and formation damage.
Fig. 10 Connectivity factor and skin versus time for different number of production wells

Fig. 11 Connectivity factor and skin versus time for different heterogeneities

Fig. 12 Connectivity factor and skin versus time for the different fluid losses
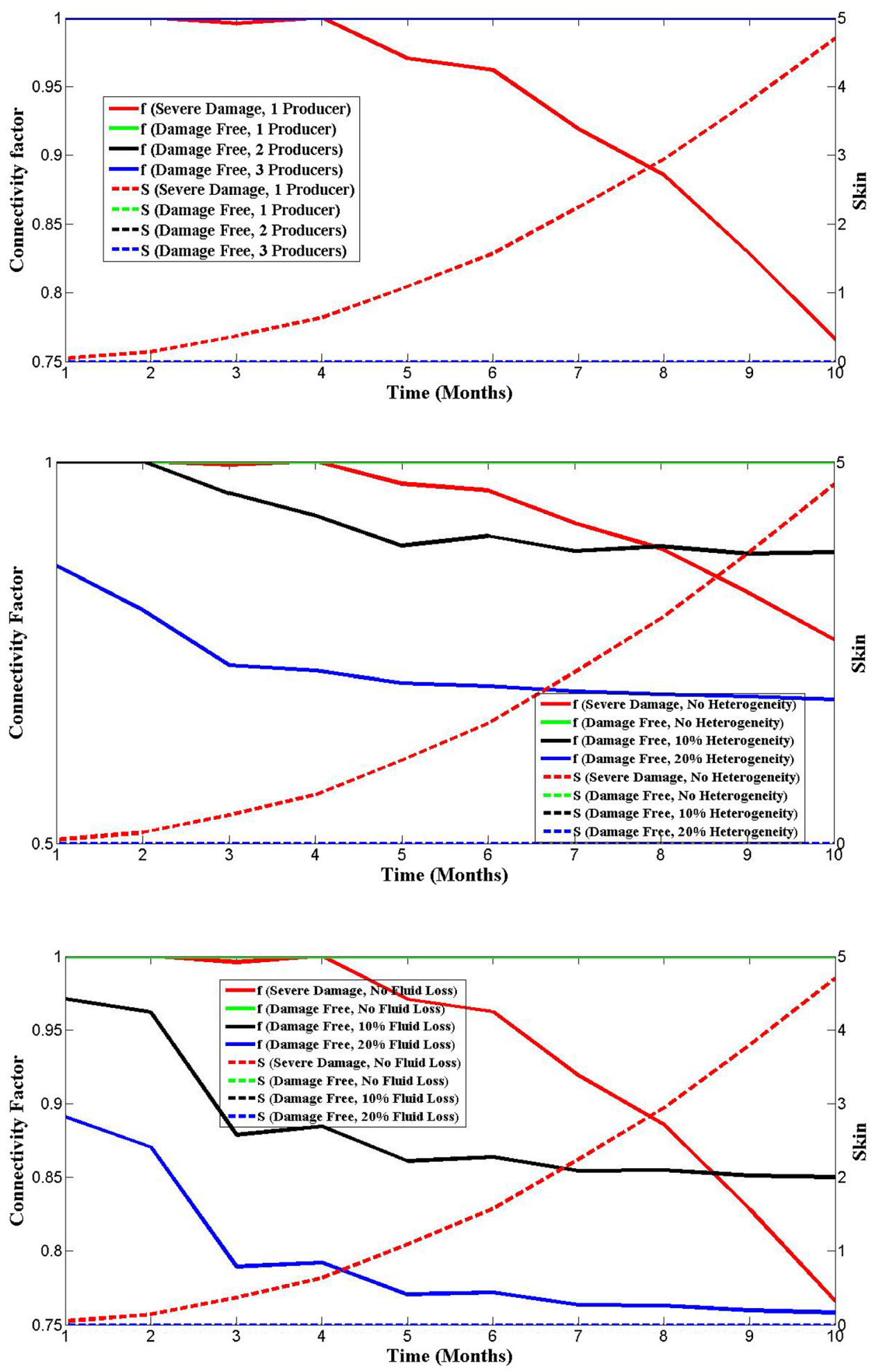

Time (Months) 


\section{Effect of fluid loss}

Existence of faults or natural fractures may lead to fluid loss in the reservoir. In this condition, a portion of the injected fluid is not directed toward the producer. For studying the effect of fluid loss on the connectivity factor of system, it was assumed that fluid loss is a time-independent process. In other words, there is a constant rate of fluid loss during the waterflooding operation. $20 \%, 10 \%$, and $0 \%$ reduction of the injection rate was selected as the representative of the fluid loss in the synthetic model. The results of that are shown in Fig. 12. As it is shown in Fig. 12, the effect of fluid loss is as same as the effect of heterogeneity. In the early times of injection, the trend of connectivity factor reduces continuously, and after enough time, it stabilizes. Like the heterogeneous cases, the stabilization of connectivity factor can be used as the criteria of difference between fluid loss and formation damage. Anyway, excessive pressure increase in the injector may cause hydraulic fracture, and a timedependent fluid loss that has not been discussed in this work.

\section{Real case}

In order to find the effect the formation damage on connectivity factor in a real case, the injection and production data of a field in southern region of Iran were used. In a real field case, the selected data must have the following properties:

- Number of the active wells must remain constant.

- The injection pressure should not change considerably (to ignore the creation of fractures).

Only 8 months of injection data with the mentioned properties were available. The unadjusted sea water with high potential of formation damage was injected into the reservoir. So, the injector was damaged considerably. The connectivity factor of the injector versus time is shown in Fig. 13. The data show that the connectivity factor of the injector has been decreased consecutively that is due to the formation damage. In Fig. 13, there is the estimated connectivity factor of the ninth month which is achieved from extrapolation.

Due to the commercial problems of an ordinary well-testing method (shutdown of well), the skin was measured only
Fig. 13 Connectivity factor for the damaged injector in the real case
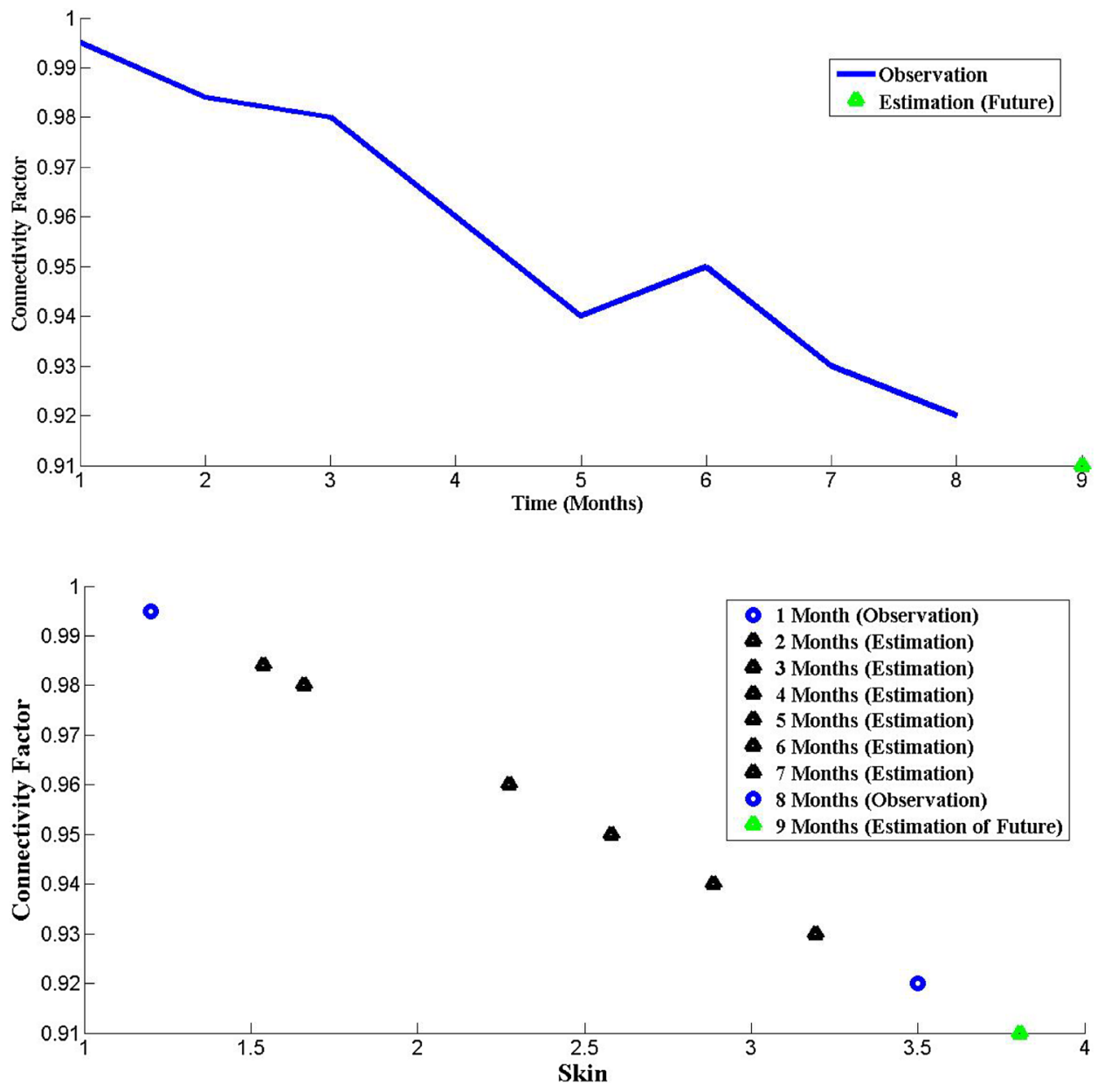

Fig. 14 Connectivity factor versus skin for the real case 
in the 1st and 8th months of injection. The amount of skins versus the connectivity factor for the real case is presented in Fig. 14. According to the linear relation of connectivity factor and skin, extrapolation has been made to find the amount of skin in other months (predictions). After the 8th month, the wellbore was acidized to remove the damages. According to Fig. 14, if the injection process was continued, the skin would be 3.8 .

\section{Conclusions}

- Continuous reduction in the connectivity factor estimated from CRM during the waterflooding operation can be considered as a representative of the formation damage in the injector.

- Heterogeneity and fluid loss have a time-independent effect on the connectivity factor of CRM, and their effect can be distinguished from the effect of formation damage.

- According to the linear relationship between connectivity factor and skin, extrapolations can be made to find the skin, without any ordinary well-testing.

Open Access This article is distributed under the terms of the Creative Commons Attribution 4.0 International License (http://creativeco mmons.org/licenses/by/4.0/), which permits unrestricted use, distribution, and reproduction in any medium, provided you give appropriate credit to the original author(s) and the source, provide a link to the Creative Commons license, and indicate if changes were made.

\section{References}

Al-Abduwani FA, Shirzadi A, van den Brock W, Currie PK (2005) Formation damage vs. solid particles deposition profile during laboratory simulated PWRI. SPE J 10(02):138-151

Alem A, Elkawafi A, Ahfir N-D, Wang H (2013) Filtration of kaolinite particles in a saturated porous medium: hydrodynamic effects. Hydrogeol J 21(3):573-586

Baghdikian SY, Sharma MM, Handy LL (1987) Flow of clay suspensions through porous media. In: SPE international symposium on oilfield chemistry. Society of Petroleum Engineers

Bedrikovetsky P, Marchesin D, Shecaira F, Souza A, Milanez P, Rezende E (2001) Characterisation of deep bed filtration system from laboratory pressure drop measurements. J Pet Sci Eng 32(2):167-177

Bedrikovetsky P, Santos A, Siqueira A, Souza A, Shecaira F (2003) A stochastic model for deep bed filtration and well impairment. In: SPE European formation damage conference. Society of Petroleum Engineers

Bedrikovetsky P, Siqueira A, de Souza A, Shecaira F (2006) Correction of basic equations for deep bed filtration with dispersion. $\mathrm{J}$ Pet Sci Eng 51(1):68-84
Bedrikovetsky P, Vaz ASL, Furtado CJ, de Souza ALS (2011) Formation damage evaluation from nonlinear skin growth during coreflooding. SPE Reserv Eval Eng 14:193-203

Bennacer L, Ahfir N-D, Bouanani A, Alem A, Wang H (2013) Suspended particles transport and deposition in saturated granular porous medium: particle size effects. Transp Porous Media 100(3):377-392

Carageorgos T, Marotti M, Bedrikovetsky P (2010) A new laboratory method for evaluation of sulphate scaling parameters from pressure measurements. SPE Reserv Eval Eng 13(03):438-448

de Holanda RW, Gildin E, Jensen JL (2018) A generalized framework for capacitance resistance models and a comparison with streamline allocation factors. J Pet Sci Eng 162:260-282

Herzig J, Leclerc D, Goff PL (1970) Flow of suspensions through porous media-application to deep filtration. Ind Eng Chem 62(5):8-35

Horne RN (1995) Modern well test analysis. Petroway Inc., Palo Alto

Iwasaki T, Slade J, Stanley WE (1937) Some notes on sand filtration [with discussion]. J Am Water Works Assoc 29(10):1591-1602

Izgec O, Kabir C (2010) Understanding reservoir connectivity in waterfloods before breakthrough. J Pet Sci Eng 75(1-2):1-12

Johnson WP, Li X, Assemi S (2007) Deposition and re-entrainment dynamics of microbes and non-biological colloids during nonperturbed transport in porous media in the presence of an energy barrier to deposition. Adv Water Resour 30(6):1432-1454

Kalantariasl A, Zeinijahromi A, Bedrikovetsky P (2014) Axi-symmetric two-phase suspension-colloidal flow in porous media during water injection. Ind Eng Chem Res 53(40):15763-15775

Kamal MS, Mahmoud M, Hanfi M, Elkatatny S, Hussein I (2019) Clay minerals damage quantification in sandstone rocks using core flooding and NMR. J Pet Explor Prod Technol 9(1):593-603

Kanimozhi B, Prakash J, Pranesh RV, Mahalingam S (2019) Numerical and experimental investigation on the effect of retrograde vaporization on fines migration and drift in porous oil reservoir: roles of phase change heat transfer and saturation. J Pet Explor Prod Technol. https://doi.org/10.1007/s13202-019-0692-z

Kaviani D, Jensen JL, Lake LW (2012) Estimation of interwell connectivity in the case of unmeasured fluctuating bottomhole pressures. J Pet Sci Eng 90:79-95

Liu X, Civan F (1996) Formation damage and filter cake buildup in laboratory core tests: modeling and model-assisted analysis. SPE Form Eval 11(01):26-30

Mahmoud M, Elkatatny S, Abdelgawad KZ (2017) Using high-and low-salinity seawater injection to maintain the oil reservoir pressure without damage. J Pet Explor Prod Technol 7(2):589-596

Mirzayev M, Riazi N, Cronkwright D, Jensen JL, Pedersen PK (2017) Determining well-to-well connectivity using a modified capacitance model, seismic, and geology for a Bakken Waterflood. J Pet Sci Eng 152:611-627

Moghadam JN, Salahshoor K, Kamp A (2011) Evaluation of waterflooding performance in heavy oil reservoirs applying capacitance-resistive model. Pet Sci Technol 29(17):1811-1824

Moghadasi J, Müller-Steinhagen H, Jamialahmadi M, Sharif A (2004) Theoretical and experimental study of particle movement and deposition in porous media during water injection. $\mathbf{J}$ Pet Sci Eng 43(3):163-181

Moreno GA (2013) Multilayer capacitance-resistance model with dynamic connectivities. J Pet Sci Eng 109:298-307

Naseri S, Moghadasi J, Jamialahmadi M (2015) Effect of temperature and calcium ion concentration on permeability reduction due to composite barium and calcium sulfate precipitation in porous media. J Nat Gas Sci Eng 22:299-312

Nwachukwu A, Jeong H, Pyrcz M, Lake LW (2018) Fast evaluation of well placements in heterogeneous reservoir models using machine learning. J Pet Sci Eng 163:463-475

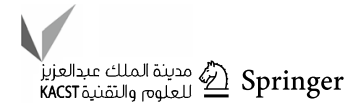


Oliveira MA, Vaz AS, Siqueira FD, Yang Y, You Z, Bedrikovetsky P (2014) Slow migration of mobilised fines during flow in reservoir rocks: laboratory study. J Pet Sci Eng 122:534-541

Pang S, Sharma M (1997) A model for predicting injectivity decline in water injection wells (Supplement to SPE 28489)

Parekh B, Kabir CS (2013) A case study of improved understanding of reservoir connectivity in an evolving waterflood with surveillance data. J Pet Sci Eng 102:1-9

Rege S, Fogler HS (1988) A network model for deep bed filtration of solid particles and emulsion drops. AIChE J 34(11):1761-1772

Rong Y, Pu W, Zhao J, Li K, Li X, Li X (2016) Experimental research of the tracer characteristic curves for fracture-cave structures in a carbonate oil and gas reservoir. J Nat Gas Sci Eng 31:417-427

Sacramento RN, Yang Y, You Z, Waldmann A, Martins AL, Vaz AS, Zitha PL, Bedrikovetsky P (2015) Deep bed and cake filtration of two-size particle suspension in porous media. J Pet Sci Eng 126:201-210

Salehian M, Çınar M (2019) Reservoir characterization using dynamic capacitance-resistance model with application to shut-in and horizontal wells. J Pet Explor Prod Technol. https://doi.org/10.1007/ s13202-019-0655-4

Santos A, Bedrikovetsky P (2006) A stochastic model for particulate suspension flow in porous media. Transp Porous Media 62(1):23-53

Sayarpour M (2008) Development and application of capacitanceresistive models to water/carbon dioxide floods. The University of Texas at Austin, Austin

Sayarpour M, Zuluaga E, Kabir CS, Lake LW (2009) The use of capacitance-resistance models for rapid estimation of waterflood performance and optimization. J Pet Sci Eng 69(3):227-238

Sharma M, Yortsos Y (1987) A network model for deep bed filtration processes. AIChE J 33(10):1644-1653

Sharma MM, Pang S, Wennberg KE, Morgenthaler L (2000) Injectivity decline in water-injection wells: an offshore Gulf of Mexico case study. SPE Prod Facil 15(01):6-13

Suleymanov AA, Abbasov AA, Guseynova DF, Babayev JI (2016) Oil reservoir waterflooding efficiency evaluation method. Pet Sci Technol 34(16):1447-1451

Todd AC, Somerville J, Scott G (1984) The application of depth of formation damage measurements in predicting water injectivity decline. In: SPE formation damage control symposium. Society of Petroleum Engineers

Vaz A, Maffra D, Carageorgos T, Bedrikovetsky P (2016) Characterisation of formation damage during reactive flows in porous media. J Nat Gas Sci Eng 34:1422-1433
Vaz A, Bedrikovetsky P, Fernandes P, Badalyan A, Carageorgos T (2017) Determining model parameters for non-linear deep-bed filtration using laboratory pressure measurements. J Pet Sci Eng 151:421-433

Wang S, Civan F (2001) Productivity decline of vertical and horizontal wells by asphaltene deposition in petroleum reservoirs. In: SPE international symposium on oilfield chemistry. Society of Petroleum Engineers

Wat R, Sorbie K, Todd A, Ping C, Ping J (1992) Kinetics of BaSO4 crystal growth and effect in formation damage. In: SPE formation damage control symposium. Society of Petroleum Engineers

Weber D, Edgar TF, Lake LW, Lasdon LS, Kawas S, Sayarpour M (2009) Improvements in capacitance-resistive modeling and optimization of large scale reservoirs. In: SPE western regional meeting. Society of Petroleum Engineers

Wong R, Mettananda D (2010) Permeability reduction in Qishn sandstone specimens due to particle suspension injection. Transp Porous Media 81(1):105

Yang H, Balhoff MT (2017) Pore-network modeling of particle retention in porous media. AIChE J 63(7):3118-3131

You Z, Osipov Y, Bedrikovetsky P, Kuzmina L (2014) Asymptotic model for deep bed filtration. Chem Eng J 258:374-385

Yuan H, Shapiro AA (2010) Modeling non-Fickian transport and hyperexponential deposition for deep bed filtration. Chem Eng J 162(3):974-988

Yuan H, Shapiro AA (2011) A mathematical model for non-monotonic deposition profiles in deep bed filtration systems. Chem Eng J 166(1):105-115

Yuan H, You Z, Shapiro A, Bedrikovetsky P (2013) Improved population balance model for straining-dominant deep bed filtration using network calculations. Chem Eng J 226:227-237

Zhang Z, Li H, Zhang D (2017) Reservoir characterization and production optimization using the ensemble-based optimization method and multi-layer capacitance-resistive models. J Pet Sci Eng 156:633-653

Publisher's Note Springer Nature remains neutral with regard to jurisdictional claims in published maps and institutional affiliations. 